\title{
Konzerneinfluss und Entkopplung vom Marktrisiko - Eine empirische Analyse der Betafaktoren bei faktischen und Vertragskonzernen
}
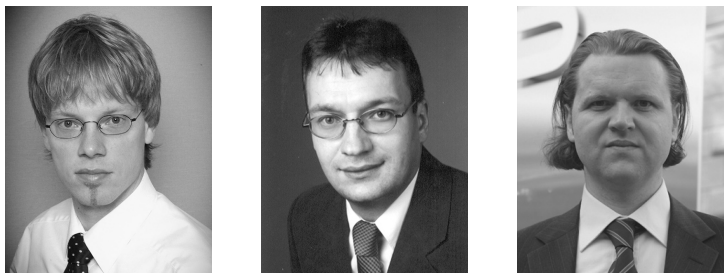

\section{Christian Brüchle, Olaf Ehrhardt, Eric Nowak}

\section{Überblick}

- Im vorliegenden Beitrag werden die Betafaktoren beherrschter Unternehmen bei faktischen und Vertragskonzern-Verhältnissen bis hin zum Squeeze-Out untersucht.

- Die Ergebnisse zeigen signifikant abnehmende Betafaktoren über verschiedene Konzernstufen.

- Bei Vertragskonzernen mit Beherrschungs- und Gewinnabführungsvertrag und Squeeze-Out-Unternehmen sinken die Betafaktoren der beherrschten Unternehmen auf Werte nahe Null.

- Die Ergebnisse sind ein Beleg für einen Marktrisiko-Entkopplungseffekt bei Unternehmen, die einem Konzerneinfluss unterliegen.

- Die Aktien von Minderheitsaktionären beherrschter Unternehmen haben somit im Durchschnitt eine niedrigere Risikoklasse als Aktien von Unternehmen im Streubesitz.

- Für die Betaschätzung zur Bewertung von Unternehmen mit einem geringen Streubesitzanteil empfehlen wir deshalb, spezielle Regressionsverfahren für unregelmäßig gehandelte Aktien (z.B. Trade-to-Trade-Verfahren) der Verwendung von Peer-GroupBetas vorzuziehen.

Keywords Valuation · beta factors · concentrated control

Eingegangen: 2. Februar 2007

JEL: G14, G31, G34

Dipl.-Oek. Christian Brüchle $(\bowtie)$

hat an der Universität Witten/Herdecke studiert. Seit 2006 ist er tätig bei Proctor \& Gamble im Bereich Finance \& Accounting. Bruechle.c@pg.com.

Prof. Dr. Olaf Ehrhardt $(\bowtie)$

ist Inhaber der Professur für Globales Finanzmanagement und International Business an der Fachhochschule Stralsund, Zur Schwedenschanze 15, D-18435 Stralsund, olaf.ehrhardt@fh-stralsund.de.

Univ.-Prof. Dr. Eric Nowak $(\bowtie)$

ist Ordinarius für Finanzmanagement und Rechnungswesen am Swiss Finance Institute an der Universität Lugano, Via Buffi 13, CH-6904 Lugano, nowake@lu.unisi.ch. 


\section{A. Einleitung}

Bei aktienrechtlichen Strukturmaßnahmen wie Unternehmensübernahmen, Fusionen, dem Abschluss von Beherrschungs- und Gewinnabführungsverträgen und Squeeze-Outs sind den Minderheitsaktionären Barabfindungsangebote zu unterbreiten, die einen Ausgleich für den Eingriff in ihre Eigentümerposition schaffen sollen. Eine Abfindung gilt nach der neueren Rechtssprechung als angemessen, wenn sie dem ausscheidenden Aktionär eine volle Entschädigung dafür verschafft, was seine Beteiligung an dem arbeitenden Unternehmen wert ist. ${ }^{1}$

Ein wesentliches Element bei der Ermittlung der Höhe des Barabfindungsangebots an die Aktionäre ist die Schätzung des wertpapierspezifischen Risikozuschlags zur Diskontierung der zukünftigen Einzahlungsüberschüsse. Bei börsennotierten Gesellschaften erfolgt diese Zuschlagsermittlung im Regelfall auf der Grundlage des Capital Asset Pricing Model (CAPM) durch eine empirische Schätzung des Betafaktors aus historischen Renditezeitreihen.

In speziellen Situationen wie der Ermittlung eines angemessenen Ausgleichs bei Abschluss eines Beherrschungsvertrages oder eines Barabfindungsangebots im Rahmen eines Squeeze-Outs bestehen Probleme bei der Schätzung dieses Betafaktors. Da der kontrollierende Großaktionär zur Durchführung eines Squeeze-Outs mindestens 95 Prozent des Grundkapitals des Unternehmens besitzen muss, werden die noch im Streubesitz befindlichen Aktien in vielen Fällen nur unregelmäßig gehandelt, analoges gilt beim Beherrschungsvertrag. Bei der Auswertung von Bewertungsgutachten ist in einigen Fällen festzustellen, dass die durch den unregelmäßigen Handel verursachten Schätzprobleme dadurch gelöst werden sollen, dass Betafaktoren von Vergleichsunternehmen (Peer Group) Verwendung finden. ${ }^{2}$ Die neuere Rechtssprechung widerspricht bei Squeeze-Out-Fällen dieser Vorgehensweise und weicht mangels Alternativen wieder auf einen pauschalen Risikozuschlag nach § 287 ZPO aus. ${ }^{3}$ Ehrhardt/Nowak (2005) bieten aus diesem Dilemma einen Ausweg, indem sie auf Verfahren verweisen, mit denen Betafaktoren auch bei unregelmäßigem Handel der Aktien geschätzt werden können. Mit diesen Verfahren erhalten sie bei Squeeze-Outs Betafaktoren nahe Null. In der Interpretation verweisen sie auf eine Entkopplung von den Marktrisiken bei Vorhandensein eines Großaktionärs. ${ }^{4}$

Der vorliegende Beitrag setzt bei der Überlegung zum Marktrisiko-Entkopplungseffekt an. ${ }^{5}$ Was Ehrhardt/Nowak (2005) in ihrer auf die Schätzung von Betafaktoren von Squeeze-out-Unternehmen fokussierten Studie nicht behandeln, ist die Tatsache, dass einige dieser Unternehmen schon über einen Beherrschungs- und Gewinnabführungsvertrag verfügen und somit Vertragsunternehmen sind. Beim Abschluss eines Beherrschungsund Gewinnabführungsvertrags erhalten die Aktionäre bereits eine Barabfindung bzw. Ausgleichszahlungen, die der ursprünglichen Aktie den Charakter einer Unternehmensanleihe gibt. Der Marktrisiko-Entkopplungseffekt kommt folglich schon bei der Errichtung eines Vertragskonzerns zum Tragen. Bei den in der vorliegenden Studie durchgeführten empirischen Analysen zeigt sich beim Übergang vom Vertragskonzern zum Squeeze-Out-Unternehmen im Durchschnitt nur eine geringe Reduzierung der Betafaktoren. Bei der Errichtung eines Vertragskonzerns ist erwartungsgemäß die deutliche und statistisch signifikante Reduzierung der Betafaktoren zu beobachten. 
Besteht ein Marktrisiko-Entkopplungseffekt, so müssten sich die Betafaktoren aber auch schon bei der Errichtung eines faktischen Konzerns signifikant verringern. Auch diese Vermutung kann im vorliegenden Beitrag empirisch bestätigt werden.

Das Hauptergebnis der Studie, dass sich die Betafaktoren mit zunehmendem Konzerneinfluss verringern und der Marktrisiko-Entkopplungseffekt somit erstmals empirisch nachgewiesen wird, hat Konsequenzen für die Methodik der Unternehmensbewertung. Die Verwendung von Betafaktoren einer Peer Group setzt nämlich voraus, dass die Vergleichsunternehmen der gleichen Risikoklasse angehören. ${ }^{6}$ Nach unseren Berechnungen haben die Aktien von Minderheitsaktionären beherrschter Unternehmen aber im Durchschnitt eine niedrigere Risikoklasse als Aktien von Unternehmen im Streubesitz.

Im nachfolgenden Abschnitt B werden die unterschiedlichen Formen bzw. Stufen der Konzerneinflussmacht aus juristischer Perspektive definiert. Abschnitt $\mathrm{C}$ beschreibt die im Kern des Beitrags stehende empirische Untersuchung der Schätzung von Betafaktoren, und der letzte Abschnitt D enthält die Schlussfolgerungen.

\section{B. Unterschiedliche Formen der Konzerneinflussmacht}

Bei der Differenzierung eines unterschiedlichen Konzerneinflussgrades ist zwischen einem faktischen Konzern und einem Vertragskonzern zu unterscheiden. Beide Formen sind unter dem rechtlichen Konstrukt des Unterordnungskonzerns gemäß § 18 Abs. 1 S. 1 AktG zu fassen.

\section{Faktischer Konzern}

Das Konstrukt des „Faktischen Konzerns“ ist zwar nicht legaldefiniert, ergibt sich aber implizit aus dem Aktiengesetz. ${ }^{7}$ Um einen faktischen Konzern handelt es sich, wenn die generellen Bedingungen eines Konzerns ( $§ 18 \mathrm{Abs} .1 \mathrm{AktG})$ gegeben sind, jedoch weder ein Beherrschungsvertrag noch eine Eingliederung vorliegen. Konkret müssen die Merkmale der Abhängigkeit sowie der einheitlichen Leitung gegeben sein. Da es gemäß $§ 18$ Abs. 1 S. 3 AktG bereits ausreicht, beim Tatbestand der Abhängigkeit zugleich von einem Konzern auszugehen, stellt die Abhängigkeit i.S.v. $§ 17$ AktG die zentrale Grundlage für die Konzernvermutung dar. ${ }^{8}$ Die Abhängigkeitsvermutung bei einer Mehrheitsbeteiligung i.S.v. $§ 17$ Abs. 2 AktG stützt sich dabei auf die Möglichkeit der mittelbaren Einflussnahme durch den Mehrheitsaktionär auf die Führung der Geschäfte der abhängigen Gesellschaft, ${ }^{9}$ „d.h. auf die Möglichkeit, kraft des Mehrheitswahlrechts die den Anteilseignern im Aufsichtsrat zustehenden Sitze mit seinen Leuten zu besetzen (vgl. § 101 AktG) und dabei sicherzustellen, dass der Aufsichtsrat auch in den Vorstand Personen beruft (vgl. § $84 \mathrm{AktG})$, die ihm genehm sind““.10

Ein Anteilsbesitz von 50 Prozent (+1 Aktie) an einer anderen Kapitalgesellschaft reicht also aus, um gemäß der Vermutungskette $\S 17$ Abs. 2 AktG und $\S 18$ Abs. 1 S. 3 AktG einen faktischen Konzern zu konstituieren. In Einzelfällen kann sogar schon eine Minderheitsbeteiligung ausreichen, um den Tatbestand der Abhängigkeit vermuten zu lassen und zwar dann, wenn die Minderheitsbeteiligung einen gleichwertigen Einfluss vermittelt wie normalerweise die Mehrheitsbeteiligung. ${ }^{11}$ 
Die bedeutendsten Rechtsfolgen des faktischen Konzerns sind in $\S \S 311$ bis 318 AktG geregelt. Gem. § 311 AktG darf die herrschende Gesellschaft (bzw. der Hauptaktionär) ihre Einflussmöglichkeiten nicht zum Nachteil der abhängigen Gesellschaft ausnutzen. Nachteilige Rechtsgeschäfte oder Maßnahmen auf Veranlassung der herrschenden Gesellschaft, welche eine Beeinträchtigung der Vermögens- und Ertragslage der abhängigen Gesellschaft darstellen, sind nur dann erlaubt, wenn die herrschende Gesellschaft i.S.v. § 311 AktG die Nachteile wieder ausgleicht bzw. einen Nachteilsausgleich rechtsverbindlich verspricht. Der von der herrschenden Gesellschaft zu erbringende Nachteilsausgleich ist nicht mit dem Verlustausgleich eines Beherrschungsvertrages gleichzusetzen, und somit verbleibt das unternehmerische Risiko zunächst beim beherrschten Unternehmen.

\section{Vertragskonzern}

Unter einem Vertragskonzern versteht man ein Konzernkonstrukt, welches sich durch den Abschluss eines Beherrschungsvertrages i.S.v. § 291 AktG ergibt. Durch den Abschluss eines Beherrschungsvertrages unterstellt eine deutsche AG oder $\mathrm{KGaA}$ die Leitung ihrer Gesellschaft einem anderen Unternehmen. Als organisatorische Rechtsfolge erwirbt hierdurch das herrschende Unternehmen ein Weisungsrecht gegenüber der abhängigen Gesellschaft gem. § 308 Abs. 1 AktG. Aus steuerlichen Gründen wird oftmals der Beherrschungsvertrag mit dem Gewinnabführungsvertrag zu einem sog. Organschaftsvertrag zusammengefasst. ${ }^{12}$ Ein Gewinnabführungsvertrag gemäß § 291 AktG liegt dann vor, wenn sich eine AG oder KGaA verpflichtet, ihren ganzen Gewinn an ein anderes Unternehmen abzuführen. Ein isolierter Gewinnabführungsvertrag allein reicht nicht aus, um einen Vertragskonzern zu konstituieren, es kann lediglich ein faktischer Konzern entstehen. ${ }^{13}$

Wie bereits beim faktischen Konzern hat der Gesetzgeber beim Abschluss von Beherrschungs- und Gewinnabführungsverträgen verschiedene Vorkehrungen zum Schutz der Minderheitsaktionäre geschaffen, die durch den Unternehmensvertrag in ihren Mitverwaltungs- und Vermögensrechten beeinträchtigt werden. Gem. § 304 AktG steht den Minderheitsaktionären im Falle eines Verbleibens im Unternehmen eine feste bzw. variable jährliche Ausgleichszahlung zu. Laut § 305 AktG hat das beherrschende Unternehmen zusätzlich die Verpflichtung, die Aktien der außenstehenden Aktionäre auf deren Verlangen gegen eine angemessene Abfindung zu erwerben.

Der faktische Konzern und der Vertragskonzern unterscheiden sich vor allem durch den unterschiedlichen Grad der Einflussmacht des herrschenden Unternehmens auf das abhängige Unternehmen. Während beim faktischen Konzern das herrschende Unternehmen nur solange Einfluss ausüben kann wie dem beherrschten Unternehmen keine (ausgleichsfähigen) Nachteile erwachsen, steht dem herrschenden Unternehmen beim Vertragskonzern ein volles Weisungsrecht gegenüber dem beherrschten Unternehmen zu. Dementsprechend gestalten sich auch die rechtlichen Konsequenzen unterschiedlich. Werden beim faktischen Konzern lediglich zugefügte Nachteile ausgeglichen, muss beim Vertragskonzern für jeglichen Verlust der beherrschten Gesellschaft aufgekommen werden. 


\section{Empirische Untersuchungen}

\section{Methodische Grundlagen}

Bei der Unternehmensbewertung von Vertragskonzernen oder bei der Ermittlung von Barabfindungsangeboten im Rahmen von Squeeze-Outs können Probleme bei der Schätzung der Betafaktoren auftreten, da die wenigen noch im Streubesitz befindlichen Aktien nur unregelmäßig gehandelt werden.

Im nachfolgenden Abschnitt wird dazu zunächst der so genannte Intervalling-Effekt genauer erläutert, der durch den unregelmäßigen Handel von Aktien verursacht wird und zu Schwankungen des Betafaktors in Abhängigkeit der Länge des Betrachtungsintervalls führt. ${ }^{14}$

Im Allgemeinen werden Betaschätzungen typischerweise durch einfache Kleinstquadrat (OLS)-Regressionen vorgenommen, die auf dem sog. Marktmodell basieren. Fisher (1966), Scholes/Williams (1977), Cohen/Maier/Schwartz/Whitcomb (1979) und Dimson (1979) stellen in ihren Analysen jedoch fest, dass bei unregelmäßig gehandelten Aktien die Betaschätzungen verzerrt sind, wenn diese mit einer OLS-Regression geschätzt werden. Deshalb sollen im Folgenden die wichtigsten Korrekturverfahren zur Bestimmung von Betafaktoren bei unregelmäßig gehandelten Aktien kurz skizziert werden.

\section{Intervalling-Effekt}

Sind die Renditen einer Aktie und des als Proxy für das Marktportfolio verwendeten Index unabhängig und identisch verteilt, so ist die Höhe des geschätzten Betafaktors nicht von der Länge des Zeitintervalls abhängig. ${ }^{15}$ die Wahl des Zeitintervalls müsste daher grundsätzlich ohne Einfluss auf den Betafaktor sein. ${ }^{16}$

Als Intervalling-Effekt bezeichnet man jedoch die Tatsache, dass die Wahl der Zeitintervalle für die Aktien- und Indexrendite (z.B. Tages-, Wochen- oder Monatsrenditen) die Höhe des Betafaktors beeinflusst. Dieser Effekt wurde vielfach empirisch untersucht und verifiziert. ${ }^{17}$ Theoretisch kann der Intervalling-Effekt auf Friktionen im Handel mit einzelnen Aktien zurückgeführt werden, durch die Messfehler bei kürzeren Renditeintervallen auftreten können. ${ }^{18}$

Für die vorliegende Untersuchung sind insbesondere Messfehler von Interesse, die aufgrund des unregelmäßigen Handels einzelner Aktien entstehen. Welche Auswirkungen diese Messfehler auf die Schätzung der Höhe des Betafaktors haben, wurde als erstes von Scholes/Williams (1977) in Anlehnung an Erkenntnisse von Fama (1965) und insbesondere Fisher (1966) untersucht. Dabei wurde festgestellt, dass trotz des täglichen Handels einige Aktien erst nach gewisser Zeit und somit nicht gleichzeitig mit dem Index auf neue Informationen reagieren. ${ }^{19}$ Aufgrund dieser Verzögerung sind nun die Kurse einiger Aktien für die Renditeberechnung ,zu alt“, was bei täglicher Renditemessung zu positiv autokorrelierten Indexrenditen führt. ${ }^{20}$ Ferner zeigen Scholes/Williams (1977), dass die beobachteten Aktienrenditen negativ autokorreliert sind, wenn die wahren Renditen (mit konstanten Varianzen und Kovarianzen) normalverteilt und die Perioden ohne Handel der Aktien unabhängig und identisch im Zeitverlauf verteilt sind..$^{21}$ Je höher der Anteil unregelmäßig gehandelter Aktien am Index ist, um so stärker weichen die intertemporalen 
Korrelationen zwischen Aktien- und Indexrenditen von Null ab, was zur Verzerrungen der OLS-Schätzer für die Betafaktoren führt. ${ }^{22}$

\section{Korrekturgleichung von Scholes/Williams (1977)}

Scholes/Williams (1977) versuchen in einem ersten Ansatz, die Effekte eines verzerrten Betafaktors durch eine Annäherung an den wahren Betawert zu eliminieren. Gemäß dem Modell von Scholes/Williams (1977) lässt sich folgende Beziehung zwischen dem geschätzten Betafaktor $\hat{\beta}_{i}$ und dem wahren Betafaktor $\beta_{i}$ ableiten: ${ }^{23}$

$$
\hat{\beta}_{i}=\beta_{i}-\left(\hat{\beta}_{i}^{-}+\hat{\beta}_{i}^{+}-2 \beta_{i} \hat{\rho}_{M}\right)
$$

(1a) mit $\quad \hat{\rho}_{M}=\frac{\operatorname{cov}\left(\widetilde{R}_{M, t} ; \widetilde{R}_{M, t-1}\right)}{\operatorname{std}\left(\widetilde{R}_{M, t}\right) \cdot \operatorname{std}\left(\widetilde{R}_{M, t-1}\right)}$

(1b) $\operatorname{mit} \quad \hat{\beta}_{i}^{-}=\frac{\operatorname{cov}\left(\widetilde{R}_{i, t} ; \widetilde{R}_{M, t-1}\right)}{\operatorname{var}\left(\widetilde{R}_{M, t-1}\right)}$

(1c) mit $\hat{\beta}_{i}^{+}=\frac{\operatorname{cov}\left(\widetilde{R}_{i, t} ; \widetilde{R}_{M, t+1}\right)}{\operatorname{var}\left(\widetilde{R}_{M, t-1}\right)}$

$\hat{\beta}_{i}^{-}=$OLS-Schätzwert für Aktie i mit der Vortagesmarktrendite als Lag-Beta,

$\hat{\beta}_{i}^{+}=$OLS-Schätzwert für Aktie i mit der Marktrendite des Folgetages als Lead-Beta,

$\hat{\rho}_{M}=$ Autokorrelationskoeffizient erster Ordnung der Marktrendite.

Bei dem Intervalling-Effekt spielen die intertemporalen Korrelationen zwischen Aktienund Indexrendite, ausgelöst durch Verzögerungen des Kurses der Aktie, eine entscheidende Rolle. ${ }^{24}$ Im Modell von Scholes/Williams (1977) werden diese durch die Verwendung von Lead- und Lag-Betafaktoren erfasst und im Korrekturverfahren berücksichtigt. ${ }^{25}$

Aus (1) leiten Scholes/Williams (1977) nun den Schätzwert für den wahren Betafaktor $a b:^{26}$

$$
\hat{\beta}_{i}^{S W}=\frac{\hat{\beta}_{i}^{+}+\hat{\beta}_{i}+\hat{\beta}_{i}^{-}}{1+2 \hat{\rho}_{M}}
$$

$\hat{\beta}_{i}=$ OLS-Schätzwert für Aktie i mit der Tagesmarktrendite,

$\hat{\beta}_{i}^{-}=$entspricht Lag-Beta (1b),

$\hat{\beta}_{i}^{+}=$entspricht Lead-Beta (1c).

In ihrem Modell gehen Scholes/Williams (1977) von Verzögerungen von einem Börsentag aus, da wie oben beschrieben, einige Aktien erst nach gewisser Zeit und somit nicht gleichzeitig mit dem Index auf neue Informationen reagieren. Ihre Betaschätzung basiert 
auf OLS-Schätzungen, welche die Aktienrendite des zu bewertenden Unternehmens mit der Vortagesmarktrendite, der Tagesmarktrendite und der Rendite des Folgetages erklärt.

\section{Korrekturgleichung von Cohen/Hawawini/Maier/Schwarz/Whitcomb (1983)}

Die theoretischen Zusammenhänge von Scholes/Williams (1977) wurden von Dimson (1979) und Cohen et al. (1983) bestätigt, welche jedoch auch Verzögerungen über einen Börsentag hinaus berücksichtigten.

Das Modell von Cohen et al. (1983) ist eine Verallgemeinerung des Schätzers $\hat{\beta}_{i}^{S W}$ von Scholes/Williams (1977) für über einen Tag hinausgehende Verzögerungen des Aktienkurses. In ihrem Modell ist die Anzahl der Betaschätzungen mit zeitlichen Lead/Lags somit variabel. Der Scholes/Williams $\hat{\beta}_{i}^{S W}$-Schätzer ist somit ein Spezialfall des $\hat{\beta}_{i}^{\text {CHMSW }}$ Schätzers von Cohen et al. (1983) bei der Anzahl von einem Lead und Lag.

$$
\hat{\beta}_{j}^{\text {CHMSW }}=\frac{b_{j}^{0}+\sum_{n=1}^{N} b_{j+n}^{0}+\sum_{n=1}^{N} b_{j-n}^{0}}{1+\sum_{n=1}^{N} b_{M+n}^{0}+\sum_{n=1}^{N} b_{M-n}^{0}}
$$

Hierbei sind $b_{j}^{0}, b_{j+n}^{0}, b_{j-n}^{0}, b_{M+n}^{0}, b_{M-n}^{0}$ OLS-Regressionsschätzer für $\beta_{j}^{0}, \beta_{j+n}^{0}, \beta_{j-n}^{0}, \beta_{M+n}^{0}, \beta_{M-n}^{0}$.

(3a) mit $\quad \beta_{j}^{0}=\operatorname{cov}\left(r_{j, t}^{0}, r_{M, t}^{0}\right) / \operatorname{var}\left(r_{M, t}^{0}\right)$

(3b) mit $\quad \beta_{M+n}^{0}=\operatorname{cov}\left(r_{M, t+n}^{0}, r_{M, t}^{0}\right) / \operatorname{var}\left(r_{M, t}^{0}\right)$

(3c) mit $\quad \beta_{M-n}^{0}=\operatorname{cov}\left(r_{M, t-n}^{0}, r_{M, t}^{0}\right) / \operatorname{var}\left(r_{M, t}^{0}\right)$

(3d) mit $\quad \beta_{j+n}^{0}=\operatorname{cov}\left(r_{j, t+n}^{0}, r_{M, t}^{0}\right) / \operatorname{var}\left(r_{M, t}^{0}\right)$

(3e) mit $\quad \beta_{j-n}^{0}=\operatorname{cov}\left(r_{j, t-n}^{0}, r_{M, t}^{0}\right) / \operatorname{var}\left(r_{M, t}^{0}\right)$

(3f) mit $\quad \beta_{j}=\operatorname{cov}\left(r_{j, t}, r_{M, t}\right) / \operatorname{var}\left(r_{M, t}\right)$

\section{Korrekturgleichung von Dimson (1979)}

Dimson (1979) verwendet in seinem Korrekturverfahren ebenfalls Lead- und Lag-Betafaktoren. Allerdings werden diese mit einem multiplen Regressionsmodell berechnet. ${ }^{27}$ Ebenso wie bei Cohen et al. (1983) kann im Dimson (1979)-Modell mit einer beliebigen Anzahl von Leads und Lags gerechnet werden.

(4) $\quad \widetilde{R}_{i, t}=\hat{\alpha}_{i}+\sum_{k=-n}^{n} \hat{\beta}_{i, k} \widetilde{R}_{m, t+k}+\widetilde{\varepsilon}_{i, t}$ 
Aus der Summe der Regressionskoeffizienten ergibt sich der $\hat{\beta}_{i}^{D}$-Betaschätzer.

$$
\hat{\beta}_{i}^{D}=\sum_{k=-n}^{n} \hat{\beta}_{i, k}
$$

Bei den nachfolgend durchgeführten empirischen Betaschätzungen wird bei den Verfahren von Cohen et al. (1983) und Dimson (1979) den Empfehlungen von Berglund/Liljeblom/ Löflund (1989) $)^{28}$ und Fowler/Rorke (1983) $)^{29}$ gefolgt und mit jeweils zwei Leads und Lags gerechnet. Berglund/Liljeblom/Löflund (1989) zeigen in ihrer Studie, dass sich mit zunehmender Anzahl von Leads und Lags die Effizienz der Schätzer verringert und eine höhere Anzahl an Leads und Lags daher aus empirischen Gründen nicht gerechtfertigt ist. ${ }^{30}$

\section{Trade-to-Trade-Verfahren}

Im Vergleich zu den zuvor vorgestellten Verfahren, die zur Eliminierung des IntervallingEffektes auf Lead- und Lag-Betafaktoren aufbauen, verwendet das Trade-to-Trade-Verfahren zur Berechnung von Renditen nur Aktienkurse von Tagen, an denen auch tatsächlich Umsätze mit der Aktie erzielt wurden. ${ }^{31}$

Da Renditen aus Perioden ohne Börsenhandel keine Berücksichtigung finden, können autokorrelierte Renditen vermieden werden. Bei diesem Verfahren werden somit alle Zeitpunkte eliminiert, an denen Marktteilnehmer über Informationen verfügen, die aufgrund eines nicht stattgefundenen Börsenhandels noch nicht in den Aktienkursen enthalten sind. Dieser Ansatz wurde erstmals von Schwert (1977), Franks/Broyles/Hecht (1977) und Marsh (1979) verwendet.

Beim Trade-to-Trade-Verfahren wird die Aktienrendite nur zwischen jenen Tagen berechnet, an denen Börsenumsätze erzielt wurden. Finden für eine Aktie z.B. an den Tagen $t=1$ und $t=5$ tatsächlich Transaktionen statt, wird eine viertägige Aktienrendite berechnet. Die entsprechende Indexrendite wird ebenfalls mit den jeweils gleichen Zeitintervallen berechnet. Alle dazwischen liegenden Tage entfallen und gehen nicht in die Berechnung ein.

Um das Trade-to-Trade-Verfahren durchführen zu können, müssen neben den für die Renditeberechnung notwendigen Daten auch die Handelsvolumina der Aktien bekannt sein. Vereinfachend können Tage mit Börsenhandel auch durch Nicht-Null-Renditen approximiert werden. ${ }^{32}$

Aufgrund der unterschiedlichen Intervalle kann das Problem auftreten, dass sich die Varianz proportional zur Länge der Residuen verhält. ${ }^{33}$ Marsh (1979) schlägt deshalb vor, die berechneten Periodenrenditen mit der Periodenlänge zu gewichten:

$$
\frac{R_{i, t_{i}}}{\sqrt{d_{t_{i}}}}=\frac{\alpha_{i p}}{\sqrt{d_{t_{i}}}}+\beta_{i p}^{T T} \cdot \frac{R_{M, t_{i}}}{\sqrt{d_{t_{i}}}}+v_{i, t_{i}} \text { mit }
$$

$R_{i, t_{i}}=$ Rendite der Aktie $\mathrm{i}$ in der Periode $\mathrm{t}_{\mathrm{i}}$ (Periode zwischen zwei aufeinanderfolgenden Transaktionen in Aktie i);

$d_{t_{i}}=$ Länge in Tagen der Periode $\mathrm{t}_{\mathrm{i}}$;

$R_{M, t_{i}}=$ Marktrendite in identischer Periode $\mathrm{t}_{\mathrm{i}}$. 


\section{Vergleich der Verfahren}

In empirischen Tests wurde die Qualität der verschiedenen Schätzverfahren untersucht. Dimson/Marsh (1983) zeigen mit Daten für den britischen Kapitalmarkt, dass die Tradeto-Trade-Regression den Korrekturverfahren vorzuziehen ist, wenn die erwarteten Renditen unregelmäßig gehandelter Aktien geschätzt werden sollen. ${ }^{34}$ Sie zeigen ferner, dass das von Cohen et al. (1983) verallgemeinerte Scholes/Williams-Verfahren nur als eine geringe Modifikation der Dimson-Methode angesehen werden kann. ${ }^{35}$ Der Unterschied zwischen beiden Verfahren ist praktisch vernachlässigbar. ${ }^{36}$ Dimson/Marsh (1983) kommen abschließend zu dem Schluss, dass Schätzprobleme in Zusammenhang mit unregelmäßig gehandelten Aktien durch Verwendung des Trade-to-Trade-Verfahrens bewältigt werden können: "[T]hin trading is indeed a serious problem. Fortunately, it is one which can be overcome by using the trade-to-trade method for estimating risk measures which are largely free from thin trading bias". ${ }^{37}$

Berglund/Liljeblom/Löflund (1989) untersuchen für den finnischen Kapitalmarkt verschiedene Korrekturverfahren zur Betaschätzung. Sie zeigen ebenfalls, dass das Trade-toTrade-Verfahren geeignet ist, bei nicht täglich gehandelten Aktien Schätzprobleme zu beheben. ${ }^{38}$ Im Ergebnis lassen sie jedoch erkennen, dass die verwendeten Korrekturverfahren im Vergleich zur Betaschätzung mit einer einfachen OLS-Regression zu ähnlichen Ergebnissen führen. ${ }^{39}$

Maynes/Ramsey (1993) untersuchen den Einfluss eines unregelmäßigen Handels in Aktien auf die Ergebnisse von Ereignisstudien. Sie überprüfen, aufbauend auf den Arbeiten von Brown/Warner (1983), die Eignung verschiedener Verfahren zur Ermittlung von Überrenditen unregelmäßig gehandelter Aktien. Dabei zeigen sie, dass lediglich die Verwendung des Trade-to-Trade-Verfahrens in Verbindung mit dem Rangsummentest von Corrado (1989) bei allen Intensitätsstufen des Handels (also auch bei unregelmäßig gehandelten Aktien) zu korrekten Renditeschätzungen und der dazugehörigen Teststatistiken führt. ${ }^{40}$ Werden hingegen andere Schätzverfahren - wie das Jumped-Return- oder das Verfahren der Renditegleichverteilung - verwendet, dann entstehen Verzerrungen bei unregelmäßigem Aktienhandel. ${ }^{41}$

Dimson/Marsh (1983), Berglund/Liljeblom/Löflund (1989) und Maynes/Ramsey (1993) empfehlen nach Auswertung der empirischen Tests zur Qualität der Regressionsschätzungen übereinstimmend die Anwendung des Trade-to-Trade-Verfahrens für die Betaschätzung von unregelmäßig gehandelten Aktien. Um auszuschließen, dass die Auswahl des Schätzverfahrens die zentralen Ergebnisse der Studie beeinflusst, werden bei den im Abschnitt III dargestellten Betaschätzungen zusätzlich die von Dimson (1979) und Cohen et al. (1993) entwickelten Verfahren für unregelmäßig gehandelte Aktien verwendet.

\section{Beschreibung des Datensatzes}

\section{Untersuchungszeitraum}

Der Datensatz enthält alle inländischen Unternehmen, deren Hauptversammlung im Zeitraum 1.1.2002 bis 31.12.2004 einen Squeeze-Out beschlossen hat. ${ }^{42}$ Die Schätzperiode für die Betafaktoren beginnt bei Vorliegen von Renditedaten am 1.1.1974, ${ }^{43}$ um die Ver- 
änderung der Betafaktoren über alle Phasen eines zunehmenden Konzerneinflusses zu ermitteln. Der Untersuchungszeitraum für die Schätzung der Betafaktoren beginnt somit frühestens am 1.1.1974 und endet spätestens am 31.12.2004.

\section{Anzahl der einbezogenen Unternehmen}

In einem ersten Schritt wurden die Unternehmen ermittelt, deren Squeeze-Out in Form einer Ad-hoc-Mitteilung auf den Internetseiten der Deutschen Gesellschaft für Ad-hocPublizität (DGAP) veröffentlicht wurde. ${ }^{44}$ Da offensichtlich vom Großteil der Unternehmen in dem untersuchten Zeitraum ein Squeeze-Out-Beschluss nicht als kursrelevante Information i.S.d. § $15 \mathrm{WpHG}$ angesehen wurde, lagen tatsächlich nur von einem geringen Teil der Squeeze-Out-Unternehmen Ad-hoc-Mitteilungen vor. Somit mussten zusätzliche Quellen zur Identifizierung von Squeeze-Out-Unternehmen herangezogen werden. Weitere Informationen wurden dem Factiva-Archiv entnommen, welches Daten von Tageszeitungen, Finanzzeitschriften, News-Tickern, von Ad-hoc-Nachrichtendienstleistern und anderen kostenpflichtigen Informationsdienstleistern enthält. ${ }^{45}$ Die restlichen Unternehmen des Datensatzes wurden mittels einer breit angelegten Presserecherche ermittelt. Letztendlich konnte eine Grundgesamtheit von 165 Unternehmen ermittelt werden, die im Zeitraum vom 01.01.2002 - 31.12.2004 einen Squeeze-Out angekündigt haben.

Aufgrund folgender Kriterien mussten 32 Unternehmen aus dem Datensatz ausgeschlossen werden:

- Der Squeeze-Out wurde angekündigt, letztendlich aber nicht von der Hauptversammlung beschlossen (3 Unternehmen).

- Die beschlussfassende Hauptversammlung zum Squeeze-Out lag außerhalb des Zeitraumes von 01.01.2002 - 31.12.2004 (8 Unternehmen).

- Der Squeeze-Out wurde abgebrochen (2 Unternehmen).

- Das Hauptversammlungsdatum des Squeeze-Out konnte nicht exakt bestimmt werden (1 Unternehmen).

- Es konnten weder Kurse, noch Aktionärsstrukturdaten ermittelt werden (8 Unternehmen).

- Es standen keine vollständigen Kursdaten zwischen dem jeweiligen Emissionsdatum und dem 31.12.2004 zur Verfügung (5 Unternehmen).

- Es standen nur ungenaue Informationen über Aktionärsstrukturverhältnisse zur Verfügung (5 Unternehmen).

Der Datensatz besteht somit insgesamt aus 133 Unternehmen.

\section{Kursdaten}

Als Grundlage für die Berechnung von Renditen für die späteren Regressionsanalysen werden Kurs- und Termindaten der einzelnen Unternehmen benötigt. Hierfür wurden die Aktienkurse der Unternehmen über den Zeitraum vom 01.01.1974 - 31.12.2004 aus dem Finanzinformationssystem Datastream gewonnen. Da über dieses System für einige Unternehmen die Kurse erst ab Oktober 1988 zur Verfügung stehen, wurden für diese Fälle die älteren Kurs- und Termindaten der Karlsruher Kapitalmarktdatenbank (KKMDB) ent- 
nommen. Dies war für solche Unternehmen der Fall, die bis zur Neuregelung der Börsensegmente 1987/88 ausschließlich im geregelten Freiverkehr notiert waren. Bei der Zusammensetzung der beiden Kursreihen (Kurse von KKMDB bis Oktober 1988 und Kurse von Datastream ab Oktober 1988) wurde darauf geachtet, dass die Kursdaten desselben Börsenplatzes Verwendung fanden, um Inkonsistenzen in den Zeitreihen zu vermeiden.

Zur Renditeberechung wurde auf mit Termindaten (Dividenden, Kapitalmaßnahmen) bereinigte Kurse zurückgegriffen, welche in Form des sog. Return Index in Datastream abrufbar sind. Bei der KKMDB stehen entsprechende Bereinigungsfaktoren zur Verfügung. Falls bei einem Unternehmen mehrere Aktien gehandelt wurden, sind nur die Kursdaten für die Stammaktien verwendet worden. Als Stellvertreter für das Marktportfolio wurde der CDAX (Performanceindex) gewählt.

\section{Datensätze für die einzelnen Konzernstufen}

\section{a) ,kein Konzern“ $(\mathrm{N}=72)$}

Die Einstufung eines Unternehmens als „kein Konzern“ erfolgt, wenn keine Mehrheitsbeteiligung durch ein anderes Unternehmen existiert. Dieses Einstufungskriterium ist eine notwendige Vereinfachung im Rahmen der empirischen Analyse, da das objektive Abklären einer faktischen Beherrschung jedes Unternehmens in der Stichprobe nicht möglich ist und eine Subjektivierung der Stichprobenselektion vermieden werden soll. Somit beinhaltet das Subsample „kein Konzern“ vermutlich auch einige tatsächlich beherrschte Unternehmen.

Eine Basisperiode ohne Konzerneinfluss lag für 72 der 133 Unternehmen vor. Die restlichen Unternehmen standen dagegen zu Beginn des Beobachtungszeitraumes am 1.01.1974 schon in einem Konzernverhältnis (entweder in einem faktischen oder Vertragskonzernverhältnis).

\section{b) „Faktischer Konzern“ ( $\mathrm{N}=\mathbf{1 2 4})$}

Bei 124 der 133 Unternehmen konnte ein Betafaktor für die Konzernstufe „Faktischer Konzern" errechnet werden. Bei den restlichen 9 Unternehmen lag entweder zu Beginn des Beobachtungszeitraums am 1.01.1974 schon die höhere Konzernstufe „Vertragskonzern“ vor (8 Unternehmen), oder es konnte aufgrund der zeitlichen Nähe der Ereignisse „Faktischer Konzern“ und „Squeeze-Out“ kein separates Beta für den Beta-Ermittlungszeitraum „Faktischer Konzern“ bestimmt werden (1 Unternehmen).

\section{c) ,Vertragskonzern“ $(\mathrm{N}=\mathbf{3 9})$}

Bei 45 der 133 Unternehmen der Stichprobe wurde im Zeitablauf vom 1.01.1974 bis 31.12.2004 ein Beherrschungs- und Gewinnabführungsvertrag bzw. ein separater Beherrschungsvertrag abgeschlossen.

Als Eintrittszeitpunkt wurde hier das Datum der beschlussfassenden Hauptversammlung gewählt. Von den 45 identifizierten Beherrschungs- und Gewinnabführungsverträgen konnte nur in 39 Fällen ein Betafaktor errechnet werden, da bei 6 Unternehmen die beschlussfassende Hauptversammlung zum Squeeze-Out und zum Beherrschungs- und Gewinnabführungsvertrag am gleichen Tag stattfand. Hier wurde bei der Betaschätzung dem stärkeren Ereignis (Squeeze-Out) der Vorrang gegeben. 


\section{d) ,Squeeze-Out“ ( $\mathrm{N}=127 / 115)$}

Da es sich bei allen 133 Unternehmen der Stichprobe um Squeeze-Out-Unternehmen handelt, liegt über den Zeitraum vom 01.01.2002 (seit Implementierung der SqueezeOut-Regelung) bis zum 31.12.2004 für alle 133 Unternehmen das Ereignis „SqueezeOut" vor.

Als Eintrittszeitpunkt wird das Datum der beschlussfassenden Hauptversammlung gewählt. Die Tatsache, dass nur 127 der 133 Squeeze-Out-Unternehmen dargestellt werden, ist auf ein früheres Delisting von 2 Unternehmen vor dem HV-Beschluss zurückzuführen, sowie auf eine Bereinigungsmaßnahme von vier extremen Ausreißer-Werten (Betafaktoren von $>3$ und $<-3$ ). Dass für die Beta-Ermittlungsstufe „Squeeze-Out“ beim Trade-toTrade-Verfahren nur 115 Betas errechnet werden können, liegt daran, dass sich bei weiteren 12 Unternehmen aufgrund zu einer zu geringen Zahl von Handelstagen mit Börsenumsätzen keine sinnvollen Regressionsergebnisse mehr ermitteln lassen.

\section{Ergebnisse}

\section{Schätzperioden der Betafaktoren für unterschiedliche Konzernstufen}

\section{a) ,kein Konzern“"}

Ein erstes Beta wird während des Zeitraums bis zum Entstehen eines faktischen oder Vertragskonzerns ermittelt.

\section{b) „Faktischer Konzern“"}

Ein zweites Beta wird zwischen dem Ereigniszeitpunkt „Faktischer Konzern“ und der nächst höheren Konzernierungsmaßnahme berechnet. Dieses Beta stellt den Einfluss der Konzernierungsmaßnahme „Faktischer Konzern“ dar. Das Ereignis „Faktischer Konzern“ tritt in dem Moment ein, in dem ein Mehrheitsaktionär mindestens 50 Prozent der Anteile eines Unternehmens erworben hat. Da die 50 Prozent Anteilsbesitz zu den Schwellenwerten gehören, die im Sinne des $\S 21 \mathrm{WpHG}$ veröffentlichungspflichtig sind, wurde in einem ersten Schritt die genauen Zeitpunkte für jedes Unternehmen über die Bundesanstalt für Finanzdienstleistungsaufsicht (BaFin) ermittelt. Da das WpHG allerdings erst im Jahr 1994 in Kraft trat und somit die BaFin über keine Datenbanken verfügt, die bis ins Jahr 1974 zurückgehen, wurde in einem zweiten Schritt auf den Hoppenstedt-Aktienführer $^{46}$ zurückgegriffen, der Aktionärsstrukturdaten aller in Deutschland notierten Unternehmen veröffentlicht. Aufgrund der Tatsache, dass der Hoppenstedt-Aktienführer nur im Ein-Jahresrhythmus aktualisiert wird, konnte nicht das exakte Datum der Überschreitung der 50-Prozent-Grenze für die jeweiligen Unternehmen identifiziert werden. In dem Jahr, in dem die Überschreitung der 50-Prozent-Grenze festgestellt werden konnte, wird sodann im Sinne einer konservativen, sicheren Ereigniszeitpunktbestimmung das Veröffentlichungsdatum des jeweiligen Hoppenstedt-Aktienführers als Ereigniszeitpunkt gewählt. Für Unternehmen, die nicht oder nur zeitweise im Hoppenstedt aufgeführt sind, wurden in einem dritten Schritt die nötigen Informationen über telefonische Anfragen direkt bei den Unternehmen eingeholt.

Da der faktische Konzern im Sinne der $\S \S 17 \mathrm{ff}$. AktG von einem Unternehmen als Mehrheitsaktionär ausgeht, wird nur der Mehrheitsbesitz durch ein Unternehmen als Er- 
eignis gewertet. Die Überschreitung der 50-Prozent-Schwelle von natürlichen Personen, wird dagegen nicht als Ereignis „Faktischer Konzern“ gewertet. Für den sehr seltenen Fall, dass die 50- Prozent-Schwelle bei einem Unternehmen mehrmals über- und wieder unterschritten wird, ist nur das Datum der letzten Überschreitung als Ereigniszeitpunkt gewählt worden. Damit verkürzt sich auch automatisch die Basisperiode „kein Konzern“, die sich dann nur über den direkt vor dem gewählten Ereignis gelegenen Zeitraum erstreckt, welcher frei von einem Konzerneinfluss ( $>50$ Prozent) ist.

\section{c), ,Vertragskonzern“"}

Ein drittes Beta ermittelt sich zwischen den Ereignissen „Beherrschungs- und Gewinnabführungsvertrag“ und „Squeeze-Out“ und repräsentiert somit die Auswirkungen eines Vertragskonzerns. Zur Bestimmung des Ereignisses „Vertragskonzern“ (Beherrschungsund Gewinnabführungsvertrag) wird ebenfalls auf den Hoppenstedt-Aktienführer zurückgegriffen.

Unter der Konzernstufe „Vertragskonzern“ sind auch isolierte Beherrschungsverträge subsumiert. Die Einflussmacht seitens des Hauptaktionärs ist bei einem isolierten Beherrschungsvertrag der Einflussmacht bei Vorliegen eines kombinierten Beherrschungsund Gewinnabführungsvertrages gleichzusetzen. ${ }^{47}$ Dies ergibt sich aus der Leitungsmacht des herrschenden Unternehmens gemäß $§ 308$ AktG.

\section{d) ,Squeeze-Out"}

Ein viertes Beta wird zwischen dem Ereignis „Hauptversammlungsbeschluss über einen Squeeze-Out“ und dem Delisting der Aktie errechnet. Für das Ereignis „Squeeze-Out“ wird ebenso mit dem Ziel eines sicheren Ereignistatbestandes wie beim Beherrschungsund Gewinnabführungsvertrag das Datum der beschlussfassenden Hauptversammlung als Ereigniszeitpunkt gewählt. Zur Ermittlung des jeweiligen Datums muss wie schon zuvor bei der Auswahl der Squeeze-Out-Unternehmen auf Ad-hoc-Nachrichtendienstleister (DGAP) sowie sonstige informationen zurückgegriffen werden (Factiva).

Tab. 1. Definition der Beta-Ermittlungszeiträume

\begin{tabular}{|c|c|c|c|}
\hline Beta- Ermittlungszeitraum & Anfangzeitpunkt & Endzeitpunkt & \\
\hline kein Konzern & $\begin{array}{l}01.01 .1974 \\
01.01 .1974 \\
01.01 .1974\end{array}$ & $\begin{array}{l}\text { Ereignis "Faktischer Konzern" } \\
\text { Ereignis "Vertragskonzern " } \\
\text { Ereignis "Squeeze-Out" }\end{array}$ & $\begin{array}{l}\text { oder } \\
\text { oder }\end{array}$ \\
\hline Faktischer Konzern & $\begin{array}{l}\text { Ereignis "Faktischer Konzern" } \\
\text { Ereignis "Faktischer Konzern" }\end{array}$ & $\begin{array}{l}\text { Ereignis "Vertragskonzern " } \\
\text { Ereignis "Squeeze-Out" }\end{array}$ & oder \\
\hline Vertragskonzern & Ereignis "Vertragskonzern" & Ereignis "Squeeze-Out" & \\
\hline Squeeze-Out & $\begin{array}{l}\text { Ereignis "Squeeze-Out" } \\
\text { Ereignis "Squeeze-Out" }\end{array}$ & $\begin{array}{l}\text { Delisting } \\
\text { 31.12.2004 }\end{array}$ & oder \\
\hline
\end{tabular}




\section{Die Höhe der Betafaktoren bei zunehmender Konzerneinflussmacht}

Für die Schätzung der Betafaktoren wird bei allen 133 Unternehmen des Datensatzes eine (i) OLS-Regression, das (ii) Trade-to-Trade-Verfahren, sowie eine (iii) Dimson- und eine (iv) CHMSW-Regression (Cohen et al. 1983) durchgeführt. Auf die Anwendung der Scholes/Williams-Regression wird verzichtet, da diese lediglich einen Sonderfall der CHMSW-Regression darstellt. Alle vier angewendeten Regressionsverfahren werden dabei für jeden der individuellen Beta-Ermittlungszeiträume pro Unternehmen durchgeführt. Für den Fall, dass ein Unternehmen über alle vier Beta-Ermittlungszeiträume (,kein Konzern“, „Faktischer Konzern“, „Vertragskonzern“, „Squeeze-Out“) verfügt, werden somit insgesamt 16 Regressionen für dieses Unternehmen durchgeführt.

In einer ersten Untersuchung werden für den gesamten (gepoolten) Datensatz die durchschnittlichen Betafaktoren für die einzelnen Konzernstufen geschätzt. Zugleich wird überprüft, ob die ermittelten Betafaktoren sich signifikant von Null unterscheiden.

Tab. 2. Schätzung der Betafaktoren für die einzelnen Konzernstufen

Der Schätzung der Betafaktoren liegen Daten von 133 Unternehmen zugrunde. Die einzelnen Stufen einer zunehmenden Konzerneinflussmacht sind wie folgt definiert: "kein Konzern" (keine Konzerneinflussmacht im aktienrechtlichen Sinne), „Faktischer Konzern“ (Einstieg eines Hauptaktionärs mit $>50 \%$ Grundkapitals), „Vertragskonzern“ (Abschluss eines Beherrschungs- und Gewinnabführungsvertrages), „Squeeze-Out“ (HVBeschluss über die Durchführung eines Squeeze-Outs). Als Test-Statistiken wurde ein T-Test zu Überprüfung der Verschiedenheit der Betafaktoren vom Wert Null sowie der nichtparametrische Kolmogorov-Smirnov-Test für die Überprüfung des Vorhandenseins einer Normalverteilung angewendet.

\begin{tabular}{|llllllll|}
\hline $\begin{array}{l}\text { Beta-Ermittlungs- } \\
\text { zeitraum }\end{array}$ & $\mathbf{N}$ & $\begin{array}{l}\text { Mittel- } \\
\text { wert }\end{array}$ & Median & Varianz & Schiefe & T-Test & $\begin{array}{l}\text { Kolmogorov- } \\
\text { Smirnov-Z }\end{array}$ \\
\hline OLS-Regression & & & & & & & \\
kein Konzern & 72 & 0,475 & 0,461 & 0,123 & 0,542 & $11,482^{* *}$ & 0,871 \\
Faktischer Konzern & 124 & 0,108 & 0,096 & 0,144 & $-3,554$ & $3,162^{* *}$ & $2,810^{* *}$ \\
Vertragskonzern & 39 & 0,023 & 0,020 & 0,001 & 0,413 & $3,935^{* *}$ & 0,517 \\
Squeeze-Out & 127 & 0,015 & 0,000 & 0,026 & 3,110 & 1,062 & $2,297^{*}$ \\
Trade-to-Trade-Verfahren & & & & & & & \\
kein Konzern & 72 & 0,564 & 0,543 & 0,118 & 0,551 & $13,938^{* *}$ & 0,707 \\
Faktischer Konzern & 124 & 0,254 & 0,195 & 0,175 & 1,940 & $6,756^{* *}$ & 1,912 \\
Vertragskonzern & 39 & 0,062 & 0,057 & 0,016 & 0,034 & $3,037^{* *}$ & 0,691 \\
Squeeze-Out & 115 & 0,044 & 0,016 & 0,080 & 1,865 & 1,667 & $2,123^{*}$ \\
Dimson-Regression & & & & & & & \\
kein Konzern & 72 & 0,611 & 0,588 & 0,189 & 0,596 & $11,936^{* *}$ & 0,676 \\
Faktischer Konzern & 124 & 0,262 & 0,159 & 0,327 & 4,325 & $5,106^{* *}$ & $2,423^{*}$ \\
Vertragskonzern & 39 & 0,054 & 0,034 & 0,011 & 0,954 & $3,210^{* *}$ & 0,709 \\
Squeeze-Out & 127 & $-0,034$ & 0,001 & 0,101 & $-2,872$ & $-1,210$ & $2,680^{* *}$ \\
CHMSW-Regression (Cohen & et al. $\mathbf{1 9 8 3 )}$ & & & & & \\
kein Konzern & 72 & 0,615 & 0,594 & 0,201 & 0,733 & $11,647^{* *}$ & 0,710 \\
Faktischer Konzern & 124 & 0,228 & 0,152 & 0,183 & 1,954 & $5,939^{* *}$ & 1,923 \\
Vertragskonzern & 39 & 0,055 & 0,036 & 0,011 & 0,937 & $3,274^{* *}$ & 0,822 \\
Squeeze-Out & 127 & $-0,016$ & 0,000 & 0,267 & $-1,342$ & $-0,358$ & $2,898^{* *}$ \\
\hline
\end{tabular}

** Signifikant verschieden mit einer Irrtumswahrscheinlichkeit von 1\%

* Signifikant verschieden mit einer Irrtumswahrscheinlichkeit von 5 \% 
Die Ergebnisse der Tabelle 2 liefern einen deutlichen Hinweis für einen MarktrisikoEntkopplungseffekt bei steigendem Konzerneinfluss, resultierend in einem kontinuierlichen Abnehmen der Betafaktoren über die Konzernierungsstufen. Für alle vier Regressionen gilt gleichermaßen, dass der durchschnittliche Betafaktor der Basisperiode „kein Konzern“, während derer es keinen Konzerneinfluss bzw. keinen Mehrheitsaktionär mit einem Stimmrechtsanteil über 50 Prozent gibt, am höchsten ist. Je nach verwendeten Verfahren ergeben sich geringfügig unterschiedliche Werte, die nahe bei 0,6 liegen. Dieser im Vergleich zu gut diversifizierten Portfolios niedrige Wert dürfte darauf zurückzuführen sein, dass ein Unternehmen häufig bereits vor dem offiziellen Erreichen der Mehrheitsbeteiligung tatsächlich beherrscht ist. Diese Tatsache ist immer dann zu vermuten, wenn aufgrund der üblichen Hauptversammlungspräsenz eine wesentliche Minderheitsbeteiligung einen gleichwertigen Einfluss wie normalerweise die Mehrheitsbeteiligung vermittelt. ${ }^{48}$

Die Beta-Ermittlungsstufe, die von dem stärksten Konzerneinflussgrad geprägt ist die Stufe „Squeeze-Out“ - zeigt für alle vier Regressionsverfahren den niedrigsten Betawert, sie weist somit den stärksten Marktrisiko-Entkopplungseffekt auf. Bemerkenswert

Tab. 3. Veränderung der Betafaktoren auf Unternehmensebene bei zunehmender Konzerneinflussmacht Die Teststatistiken basieren auf dem T-Test für abhängige Stichproben und dem Wilcoxon-Rangtest. Für die Untersuchung konnten nur die Unternehmen des Datensatzes von 133 Unternehmen verwendet werden, welche die getesteten Konzernstufen aufweisen. Die einzelnen Stufen einer zunehmenden Konzerneinflussmacht sind wie folgt definiert: "kein Konzern" (keine Konzerneinflussmacht im aktienrechtlichen Sinne), „Faktischer Konzern“ (Einstieg eines Hauptaktionärs mit > 50\% Grundkapitals), ,Vertragskonzern“ (Abschluss eines Beherrschungs- und Gewinnabführungsvertrages), „Squeeze-Out“ (HV-Beschluss über die Durchführung eines Squeeze-Outs).

\begin{tabular}{|lllll|}
\hline Beta- Ermittlungsstufen & N & $\begin{array}{l}\text { Differenz } \\
\text { Mittelwert }\end{array}$ & T-Test & Wilcoxon - Z \\
\hline OLS-Regression & & & & \\
kein Konzern - Faktischer Konzern & 68 & 0,432 & $8,922^{* *}$ & $6,947^{* *}$ \\
Faktischer Konzern - Vertragskonzern & 31 & 0,108 & 0,878 & $3,194^{* *}$ \\
Vertragskonzern - Squeeze-Out & 38 & 0,020 & 0,584 & 1,704 \\
Trade-to-Trade-Verfahren & & & & \\
kein Konzern - Faktischer Konzern & 68 & 0,434 & $7,634^{* *}$ & $6,251^{* *}$ \\
Faktischer Konzern - Vertragskonzern & 31 & 0,409 & $3,971^{* *}$ & $3,861^{* *}$ \\
Vertragskonzern - Squeeze-Out & 35 & 0,021 & 0,425 & 1,196 \\
Dimson-Regression & & & & \\
kein Konzern - Faktischer Konzern & 68 & 0,467 & $5,548^{* *}$ & $6,251^{* *}$ \\
Faktischer Konzern - Vertragskonzern & 31 & 0,582 & $3,488^{* *}$ & $4,488^{* *}$ \\
Vertragskonzern - Squeeze-Out & 38 & 0,163 & $2,389 *$ & $3,952^{* *}$ \\
CHMSW-Regression (Cohen et al. 1983) & & & & \\
kein Konzern - Faktischer Konzern & 68 & 0,519 & $7,972 * *$ & $6,477^{* *}$ \\
Faktischer Konzern - Vertragskonzern & 31 & 0,467 & $4,430^{* *}$ & $4,409 * *$ \\
Vertragskonzern - Squeeze-Out & 38 & 0,038 & 0,327 & $2,487^{*}$ \\
\hline
\end{tabular}

** Signifikant verschieden mit einer Irrtumswahrscheinlichkeit von 1\%

* Signifikant verschieden mit einer Irrtumswahrscheinlichkeit von $5 \%$ 
ist die Tatsache, dass alle vier Verfahren über die vier Beta-Ermittlungsstufen relativ ähnliche absolute Mittelwerte für die Beta-Werte generieren. Hier lässt sich bereits feststellen, dass die Korrekturverfahren (wie erwartet) die OLS-Betas nach oben korrigieren, wobei die Differenz zu den OLS-Werten aber relativ gering ist. ${ }^{49}$

In einem zweiten Schritt wird überprüft, ob die Betafaktoren auf Unternehmensebene (abhängige Stichproben) für die jeweiligen Beta-Ermittlungsstufen („kein Konzern“, „Faktischer Konzern“, „Vertragskonzern“, „Squeeze-Out“) signifikant verschieden sind und unterschiedliche Konzerneinflussgrade zu signifikant anderen Betafaktoren führen (siehe Tabelle 3).

Beide Teststatistiken, der T-Test für abhängige Stichproben sowie der nichtparametrische Wilcoxon-Test, liefern im Prinzip dasselbe Ergebnis: Es liegen signifikante Unterschiede in der Höhe der Betafaktoren bei den Übergängen „kein Konzern“ - „Faktischer Konzern“" und „Faktischer Konzern“- „Vertragskonzern“"vor. Da die Betafaktoren der drei genannten Beta-Ermittlungsstufen zusätzlich über die drei Konzernierungstufen hinweg fallen (siehe Mittelwerte aus Tabelle 2), kann die Schlussfolgerung gezogen werden, dass ein Marktrisiko-Entkopplungseffekt bei zunehmender Konzerneinflussmacht besteht.

Dass für die Kombination „Vertragskonzern“ - „Squeeze-Out“ keine signifikanten Unterschiede mehr festgestellt werden können, widerspricht dem festgestellten MarktrisikoEntkopplungseffekt keineswegs. Aufgrund der Tatsache, dass ein Beherrschungs- und Gewinnabführungsvertrag bereits eine sehr hohe Einflussmacht bedeutet, ist die Differenz gegenüber einer 100\%igen Einflussmacht, welche nach einem Squeeze-Out-Beschluss vorliegt, nur sehr gering.

\section{Vergleich der Verfahren}

Im Folgenden wird nun überprüft, ob die Korrekturverfahren ähnliche absolute Betawerte wie die in der Praxis standardmäßig verwendete OLS-Regresssion liefern. Die nachstehende Tabelle 4 zeigt die Ergebnisse der verwendeten Test-Statistiken.

Nach den vorliegenden Ergebnissen unterscheiden sich die Betafaktoren auf Basis von Verfahren, die speziell für unregelmäßig gehandelte Aktien entwickelt wurden, signifikant von den Werten der OLS-Schätzungen. Dies gilt mit Ausnahme der vierten BetaErmittlungsstufe „Squeeze-Out“. Bei unregelmäßig gehandelten Aktien werden die Betafaktoren mit einer OLS-Regression systematisch niedriger geschätzt. Der Vergleich des Trade-to-Trade-Verfahrens mit den beiden anderen Korrekturverfahren nach Dimson (1979) und Cohen et al. (1983) führt hingegen zu keinen signifikant unterschiedlichen Betafaktoren.

\section{Robustheit der Ergebnisse}

\section{a) „Out-of-sample“-Test}

Der in diesem Robustheits-Test verwendete Datensatz setzt sich aus Unternehmen zusammen, die zum 1.11.2004 gemäß der Information des Hoppenstedt-Aktienführers über einen Beherrschungs- und Gewinnabführungsvertrag verfügen, bis zum 31.12.2004 aber keinen Squeeze-Out angekündigt haben. Damit ist dieser Datensatz unabhängig vom unternehmensspezifischen Ereignis „Squeeze-Out“. 
Tab. 4. Vergleich der Schätzverfahren

Mittelwertvergleich der unter Verwendung von OLS-, Trade-to-trade-, Dimson (1979)- und CHMSW (1983)Regression (Cohen et al. 1983) geschätzten Betafaktoren mit dem T-Test für abhängige Stichproben und dem nichtparametrischen Wilcoxon-Test. Die einzelnen Stufen einer zunehmenden Konzerneinflussmacht sind wie folgt definiert: "kein Konzern“ (keine Konzerneinflussmacht im aktienrechtlichen Sinne), „Faktischer Konzern“ (Einstieg eines Hauptaktionärs mit $>50 \%$ Grundkapitals), „Vertragskonzern“ (Abschluss eines Beherrschungs- und Gewinnabführungsvertrages), ,Squeeze-Out“(HV-Beschluss über die Durchführung eines SqueezeOuts).

\begin{tabular}{|c|c|c|c|c|}
\hline Beta- Ermittlungszeitraum & $\mathbf{N}$ & $\begin{array}{l}\text { Differenz } \\
\text { Mittelwerte }\end{array}$ & T- Test & Wilcoxon - Z \\
\hline \multicolumn{5}{|c|}{ OLS-Regression vs. Trade-to-Trade-Verfahren } \\
\hline kein Konzern & 72 & $-0,089$ & $-10,748 * *$ & $-7,144 * *$ \\
\hline Faktischer Konzern & 124 & $-0,146$ & $-2,802 * *$ & $-6,369 * *$ \\
\hline Vertragskonzern & 39 & $-0,040$ & $-2,351 *$ & $-2,582 * *$ \\
\hline Squeeze-Out & 115 & $-0,023$ & $-1,227$ & $-0,857$ \\
\hline \multicolumn{5}{|c|}{ OLS-Regression vs. Dimson-Regression } \\
\hline kein Konzern & 72 & $-0,137$ & $-4,719 * *$ & $-4,955 * *$ \\
\hline Faktischer Konzern & 124 & $-0,154$ & $-2,252 *$ & $-5,237 * *$ \\
\hline Vertragskonzern. & 39 & $-0,031$ & $-2,350 *$ & $-2,456^{*}$ \\
\hline Squeeze-Out & 127 & 0,049 & 1,401 & 0,04 \\
\hline \multicolumn{5}{|c|}{ OLS-Regression vs. CHMSW-Regression } \\
\hline kein Konzern & 72 & $-0,140$ & $-4,625 * *$ & $-4,938 * *$ \\
\hline Faktischer Konzern & 124 & $-0,121$ & $-2,244 *$ & $-4,823 * *$ \\
\hline Vertragskonzern & 39 & $-0,032$ & $-2,414 *$ & $-2,679 * *$ \\
\hline Squeeze-Out & 127 & 0,032 & 0,616 & 0,107 \\
\hline \multicolumn{5}{|c|}{ Trade-to-Trade-Verfahren gegen Dimson-Regression } \\
\hline kein Konzern & 72 & $-0,048$ & $-1,676$ & $-0,909$ \\
\hline Faktischer Konzern & 124 & $-0,009$ & $-0,266$ & $-0,392$ \\
\hline Vertragskonzern & 39 & 0,009 & 0,430 & 0,321 \\
\hline Squeeze-Out & 115 & 0,084 & $2,042^{*}$ & 1,418 \\
\hline \multicolumn{5}{|c|}{ Trade-to-Trade-Verfahren gegen CHMSW-Regression } \\
\hline kein Konzern & 72 & $-0,051$ & $-1,700$ & $-0,797$ \\
\hline Faktischer Konzern & 124 & 0,025 & 1,007 & 0,711 \\
\hline Vertragskonzern & 39 & 0,008 & 0,391 & 0,265 \\
\hline Squeeze-Out & 115 & 0,062 & 1,078 & 1,091 \\
\hline
\end{tabular}

** Signifikant verschieden mit einer Irrtumswahrscheinlichkeit von 1\%

* Signifikant verschieden mit einer Irrtumswahrscheinlichkeit von $5 \%$

Dieser Datensatz enthält 32 Unternehmen, von denen bei 24 die relevanten Kursdaten und Ereignisinformationen erfasst werden können. Für die Konzernstufen „Faktischer Konzern“ und „Vertragskonzern“"werden die Betafaktoren für 17 bzw. 24 Unternehmen mit dem OLS-Verfahren und den drei verwendeten Verfahren zur Renditeschätzung für unregelmäßig gehandelte Aktien geschätzt. Die durchgeführten Mittelwertvergleiche (T-Test, Mann-Whitney-Test) zeigen keine signifikanten Unterschiede zu den Betafaktoren des ursprünglichen Datensatzes. ${ }^{50}$ 


\section{b) Unterschiedliche Periodenlängen bei der Betaschätzung}

Für diesen zweiten Robustheits-Test wird die Periodenlänge für die Schätzung der Betafaktoren auf maximal drei Jahre nach dem Ereignis begrenzt, um auszuschließen, dass gegebenenfalls unterschiedliche Periodenlängen für die einzelnen Beta-Ermittlungsstufen die Qualität der Betaschätzung auf systematische Weise beeinflussen.

Die Ergebnisse bestätigen erneut die systematische Reduzierung der Betafaktoren mit zunehmendem Konzerneinfluss. Des Weiteren können die in der Tabelle 3 dargestellten signifikanten Mittelwertunterschiede der Betafaktoren zwischen den einzelnen Konzernstufen verifiziert werden.

Ein Mittelwertvergleich der Betafaktoren mit denen in der Tabelle 2 ausgewiesenen Werten zeigt bei Anwendung des OLS-Verfahrens für die Konzernstufen „Faktischer Konzern“ und „Vertragskonzern“ signifikant höhere Betafaktoren. Beim Trade-to-TradeVerfahren ergeben sich hingegen keine signifikanten Mittelwertunterschiede.

\section{Schlussfolgerungen}

Im Rahmen unserer empirischen Untersuchung kann ein Marktrisiko-Entkopplungseffekt bei im Streubesitz befindlichen Aktien von Unternehmen, die einem Konzerneinfluss unterliegen, konstatiert werden. Dabei ist zu beobachten, dass mit zunehmendem Konzerneinfluss ein steigender Marktrisiko-Entkopplungseffekt einhergeht, welcher durch signifikant abnehmende Betafaktoren über die verschiedenen Konzernstufen nachgewiesen werden kann. So werden bei faktischen Konzernen statistisch signifikant geringere Betafaktoren (im Durchschnitt über alle Verfahren Betas von 0,25 vs. 0,60 ) als bei Unternehmen ohne Mehrheitsaktionär festgestellt. Bei Abschluss eines Beherrschungs- und Gewinnabführungsvertrages sinken die Betafaktoren bei den betroffenen Aktien im Durchschnitt nahezu auf Null $(0,06)$ und liegen signifikant unter den Betawerten von faktischen Konzernen.

Beim Übergang von einem Vertragskonzern zu einem Squeeze-Out-Unternehmen ist hingegen nur noch eine geringe Reduzierung der Betafaktoren festzustellen. Da die Aktionäre bereits beim Abschluss eines Beherrschungs- und Gewinnabführungsvertrags im Regelfall feste Ausgleichszahlungen erhalten, besitzen die im Streubesitz befindlichen Aktien bereits den Charakter eines festverzinslichen Wertpapiers. Der Marktrisiko-Entkopplungseffekt entfaltet seine ökonomische Wirkung bereits bei der Errichtung des Vertragskonzerns. ${ }^{51}$

Bei der Schätzung von Betafaktoren für die Unternehmenswertermittlung im Rahmen der aktienrechtlich vorgeschriebenen Barabfindungsangebote kann das Problem auftreten, dass die Aktien aufgrund des geringen Streubesitzanteils nur unregelmäßig gehandelt werden. Speziell für den Fall unregelmäßig gehandelter Aktien wurden mit dem Trade-totrade-Verfahren und den sogenannten Korrekturverfahren Methoden der Betaschätzung entwickelt, welche die bei unregelmäßigem Handel entstehenden statistischen Verzerrungen der Regressionsergebnisse beseitigen. Werden hingegen Peer-Group-Betas bei der Bewertung von faktischen und Vertragskonzernen (einschließlich bei Squeeze-Out-Bewertungen) verwendet, so werden die Betafaktoren zu hoch ausgewiesen, wenn der in dieser Arbeit nachgewiesene Marktrisiko-Entkopplungseffekt unberücksichtigt bleibt. Die Folge wären zu niedrige Barabfindungsangebote an die Minderheitsaktionäre. 


\section{Anmerkungen}

1 BverfGE 14, 263,284; BGH NJW 2003, 3272; OLG Düsseldorf, AG 1990, 397; BayObLG, AG 2006, 41,42 .

2 Vgl. BDO Wirtschaftsprüfungsgesellschaft, 2005, S. 18, mit Verweis auf ein ,unzureichendes Bestimmtheitsmaß des Beta-Faktors"; TPA Horwath Wirtschaftsprüfung GmbH, 2007, S. 9.; BFJM GmbH Wirtschaftsprüfungsgesellschaft, 2007, S. 26 mit Verweis auf ,nahe Null bzw. statistisch nicht signifikante Betafaktoren; Warth \& Klein, 2007a, S. 46; ders., 2007b, S.42; ders., 2006a, S. 26; ders., 2006b, S. 32, lehnen die Verwendung eigener Betas sogar ohne Begründung aus ,grundsätzlichen Überlegungen“ ab (peer group ist immer zu bevorzugen). Popp, 2006, S. 446, bestätigt diese Praxis.

3 Vgl. LG Frankfurt/M., Beschluss vom 2.5. 2006 - 3-05 O 153/04. Weitere Judikate sind LG Hamburg, Beschluss vom 3.4.2007 - 414 O 26/97 („Ölmühle“), LG Dortmund, Beschluss vom 19.3.2007 - 18 AktE 5/03 („Grohe“) und ÖLG München, Beschluss vom 19.10.2006 - 31 Wx 092/05 („Fränkisches Überlandwerk“). Eine ausführlichere Darstellung und Bewertung für diese Linie der Rechtssprechung siehe Knoll, 2006.

4 Die Vermutung eines bestehenden Marktrisiko-Entkopplungseffekt findet sich erstmals bei Knoll (2005).

5 Uns sind z.Zt. keine weiteren deutsch- oder englischsprachigen Publikationen mit gleicher Fragestellung bekannt, die dieses Thema empirisch behandeln.

6 Diese Idee geht auf Modigliani/Miller (1958) zurück. Mit dem CAPM, welches von einem linearen Zusammenhang zwischen Rendite und Risiko ausgeht, ist es gelungen, auf das diskrete Konzept der Risikoklasse zu verzichten.

$7 \S 16$ ff. AktG.

8 Vgl. Raiser, 2001, S. 818.

9 Emmerich/Habersack, 2003, S. 42.

10 Raiser, 2001, S. 819.

11 Ders., S. 820: Im Fall Veba/Gelsenberg hat der BGH..."mit Recht eine Abhängigkeit der VEBA vom Bund angenommen, obwohl dieser nur über $43,7 \%$ der Aktien verfügte, weil angesichts der üblichen Hauptversammlungspräsenz davon auszugehen war, dass dies für Mehrheitsentscheidungen ausreicht.“

12 Körperschaftsteuerliche Organschaft gem. § $14 \mathrm{KStG}$.

13 Vgl. Wirtschaftsprüfer Handbuch, 2000, S. 1952.

14 Vgl. Zimmermann, 1997, S. 119.

15 Ders., S. 100.

16 Vgl. Baetge/Krause, 1994, S. 441.

17 Vgl. Frantzmann, 1990, S. 71-74.

18 Vgl. Zimmermann, 1997, S. 100.

19 Vgl. Fisher (1966), S. 198-199 und 205-206.

20 Vgl. Zimmermann, S. 101.

21 Vgl. Scholes/Williams, 1977, S. 315.

22 Vgl. Zimmermann, 1997, S. 101.

23 Vgl. Scholes/Williams, 1977, S. 315.

24 Vgl. Zimmermann, 1997, S. 124.

25 Vgl. Scholes/Williams, 1977, S. 315.

26 Ders., S. 317.

27 Vgl. Dimson, 1979, S. 203 f.

28 Vgl. Berglund/Liljeblom/Löflund, 1989, S. 61.

29 Vgl. Fowler/Rorke, 1983, S.281-282.

30 Vgl. Berglund/Liljeblom/Löflund, 1989, S. 61.

31 Vgl. Zimmermann, 1997, S. 120.

32 Ders, S. 121.

33 Ders., S. 121.

34 Dimson/Marsh, 1983, S. 756-757.

35 Ders., S. 756.

36 Ders., S. 756. Diese Mutmaßung von Roll (1981) wurde analytisch und empirisch von Dimson (1982) bestätigt.

37 Ders., S. 780.

38 Vgl. Berglund/Liljeblom/Löflund, 1989, S. 61.

39 Ders., S. 61. 
40 Vgl. Maynes/Ramsey, 1993, S. 146.

41 Ders., S. 156.

42 Der Beginn des Erhebungszeitraums markiert das Inkrafttreten des Wertpapierübernahmegesetzes (WpÜG).

43 Beginn der Datenzeitreihen der Karlsruher Kapitalmarktdatenbank

44 Quelle: www.dgap.de.

45 Quelle: www.factiva.com.

46 Hoppenstedt-Aktienführer, Darmstadt, 1996-2004. Davor Saling, A., Aktienführer, Darmstadt, 1974-1995.

47 Ein separater Gewinnabführungsvertrag wurde dabei nicht unter das Ereignis „Vertragskonzern“ subsumiert, da diese Art von Vertrag nach herrschender Meinung nur ein faktisches Konzernverhältnis konstituiert, vgl. Raiser (2001), S. 806.

48 Die Hauptversammlungspräsenz der DAX 30-Unternehmen lag im Zeitraum zwischen 1998 und 2003 bei durchschnittlich 49\% und schwankt zwischen 23\% (Adidas-Salomon) und 79\% (Deutsche Post), vgl. Deutsche Schutzvereinigung für Wertpapierbesitz e.V., HV-Präsenzen der DAX 30-Unternehmen(1998-2003).

49 Diese Erwartung resultiert aus der Tatsache, dass es sich bei den hier verwendeten Unternehmen, um unregelmäßig gehandelte Werte handelt (zu mindestens vor und während der Squeeze-Out Phase), zu der Wirkung der Korrekturverfahren bei unregelmäßig gehandelten Aktien siehe Zimmermann, 1997, S. 119 ff.

50 Aufgrund der geringen Zahl von Beobachtungen für die Konzernstufe „kein Konzern“ (N=8) konnte hierfür kein Mittelwertvergleich durchgeführt werden.

51 Dies verifiziert die These von Knoll (2005, S. 176) nach der ein dominierender Grossaktionär die Leitung der Geschäfte im Hinblick auf den Zweck beeinflussen wird, ,den er mit seinem Einstieg verfolgt hat. Deshalb entkoppelt sich aber die Risikolage weitgehend von Marktrisiken, die für das CAPM charakteristisch sind. Folglich überrascht es kaum, dass empirische Beta-Werte, die über den Zeitraum ermittelt werden, in denen der für den Entschädigungszeitpunkt relevante Einfluss des Hauptaktionärs bestand, gering oder sogar negativ ausfallen, während der Durchschnittswert des Gesamtmarkts bei eins liegt.“

\section{Literatur}

Baetge, J., Krause, C., Die Berücksichtigung des Risikos bei der Unternehmensbewertung, in: Betriebswirtschaftliche Forschung und Praxis, 1994, S. 433-456.

BDO Wirtschaftsprüfungsgesellschaft, Angemessenheit der Barabfindung für die Kromschröder AG, 27.10. 2005, URL: www.kromschroeder.de/fileadmin/ kromschroeder/investor_relations/pdf/Pruefungsbericht_ Barabfindung.pdf [Zugang 02.02.2006]

Berglund, T., Liljeblom, E., Löflund, A., Estimating Betas on Daily Data for a Small Stock Market, in: Journal of Banking and Finance, 1989, S. 41-64.

BFJM Bachem Fervers Janssen Mehrhoff GmbH Wirtschaftsprüfungsgesellschaft, Bericht über die Prüfung des Beherrschungs- und Gewinnabführungsvertrages zwischen der CHG Communications Holding GmbH \& Co. KG, München und der CYCOS Aktiengesellschaft, Alsdorf gemäss § 293b AktG, 20. März 2007.

Brown, S., Warner, J., Using Daily stock returns - The case of event studies, in: Journal of Financial Economics, 1985, S. 3-31.

Cohen, K. J., Hawawini, G. A., Maier, S. F., Schwartz, R. A., Whitcomb, D. K., Friction in the Trading Process and the Estimation of Systematic Risk, in: Journal of Financial Economics, 1983, S. 263-278.

Cohen, K. J., Maier, S. F., Schwartz, R. A., Whitcomb, D. K., On the Existence of Serial Correlation in an Efficent Securities Market, in: TIMS Studies In The Management Sciences, 1979, S. 151-168.

Corrado, C.J., A nonparametric test for abnormal security-price performance in event studies, in: Journal of Financial Economics, 1989, S. 385-395.

Dimson, E., Risk Measurement when Shares are Subject to Infrequent Trading, in: Journal of Financial Economies, 1979, S. 197-226.

Dimson, E., Friction in the Trading Process and Risk Measurement: A Note, Working Paper, 1982, London Business School

Dimson, E., Marsh, P., The Stability of Risk Measures and the Problem of Thin Trading, in: Journal of Finance, 1983, S. 753-783.

Ehrhardt, O., Nowak, E., Viel Lärm um Nichts? - Zur (Ir)Relevanz der Risikoprämie für Unternehmensbewertungen im Rahmen von Squeeze-Outs, in: Die Aktiengesellschaft, Sonderheft, 2005, S. 6.

Emmerich, V., Habersack, M., Aktien- und GmbH-Konzernrecht: Kommentar, 4. Aufl., München, 2003.

Fama, E., Tomorrow on the New York Stock Exchange, in: Journal of Business, 1965, S. 285-299. 
Fisher, L., Some New Stock-Market Indexes, in: Journal of Business, 1966, S. 191-225.

Fowler, D. J., Rorke, H. C., Risk Measurement When Shares are subjected to Infrequent Trading: Comment, in Journal of Financial Economics, 1983, S.279-283.

Franks, J. R., Broyles, J. E., Hecht, M. J., An industry study of the profitability of mergers in the United Kingdom, in: Journal of Finance, 1977, S. 1513-1525.

Frantzmann, H. J., Zur Messung des Marktrisikos deutscher Aktien, in: Zeitschrift für betriebswirtschaftliche Forschung, 1990, 42/1, S. 67-83.

Knoll, L. , Die Ermittlung des Beta-Faktors im CAPM bei aktienrechtlichen Zwangsabfindungen, in: Unternehmensbewertung und Management (UM), 6/2005, S. $174 \mathrm{ff}$.

Knoll, L., Risikozuschlag und objektivierter Unternehmenswert im aktienrechtlichen Spruchverfahren: Einmal CAPM und zurück, in: ZSteu, 2006, 468-477.

Marsh, P., Equity Rights Issues and the Efficiency of the UK Stock Market, in: Journal of Finance, 1979, S. 839-862.

Maynes, E., Ramsey, J., Conducting Event Studies with Thinly Traded Stocks, in: Journal of Banking and Finance, 1993, S. 145-157.

Modigliani, F., Miller, M.H., The Cost of Capital, Corporate Finance, and the Theory of Investment, in: American Economic Review, 1958, 261-297.

Popp, M. (2006), Squeeze-out-Abfindung bei Beherrschungs- und Gewinnabführungsverträgen, in: Die Wirtschaftsprüfung, 7/2006, 436-448.

Raiser, T., Recht der Kapitalgesellschaften: ein Handbuch für Praxis und Wissenschaft; Aktiengesellschaft, Kommanditgesellschaft auf Aktien, Gesellschaft mit beschränkter Haftung \& Co., Umwandlungsrecht, Konzernrecht, internationales Gesellschaftsrecht, 3. Aufl., München, 2001.

Roll, R. (1981), A Possible Explanation of the Small Firm Effect, in: Journal of Finance 36, S. 879-888

Scholes, M., Williams, J., Estimating Betas From Nonsynchronous Data, in: Journal of Financial Economies, 1977, S. 309-327.

Schwert, G. W., Stock Exchange Seats as Capital Assets, in: Journal of Financial Economics, 1977, S. 51-78.

TPA Horwath, Bericht über die Prüfung gemäß § 3 Abs 2 GesAusG der Bank Austria Creditanstalt AG, 2007.

Warth \& Klein, Bericht über die Prüfung der Angemessenheit der Barabfindung für die beabsichtigte Übertragung der Aktien der Aktionäre der Degussa AG, Düsseldorf, auf die RAG Projektgesellschaft mbH, Essen, 18.04.2006a.

Warth \& Klein, Bericht über die Prüfung des Beherrschungsvertrags zwischen der SINGULUS TECHNOLOGIES Beteiligungs GmbH, Kahl am Main, und der STEAG HamaTech Aktiengesellschaft, Sternenfels, gemäß $§ 293$ b Abs. 1 AktG, 21.04.2006b.

Warth \& Klein, Bericht über die Prüfung des Beherrschungs- und Gewinnabführungsvertrages zwischen der UCB SP GmbH, Monheim, und der Schwarz Pharma Aktiengesellschaft, Monheim, gemäß $§ 293$ b Abs. 1 AktG, 23.03.2007a.

Warth \& Klein, Bericht über die Prüfung der Angemessenheit der Barabfindung für die beabsichtigte Übertragung der Aktien der Aktionäre der Bayerische Hypo- und Vereinsbank Aktiengesellschaft, München, auf die UniCredito Italiano Società per Azioni, Genua/Italien, 14.05.2007b.

Wirtschaftsprüfer Handbuch, 12. Auflage, Düsseldorf, 2000.

Zimmermann, P., Schätzung und Prognose von Betawerten, Bad Soden/Ts.:Uhlenbruch, 1997, S. 18 ff. 


\section{Konzerneinfluss und Entkopplung vom Marktrisiko - Eine empirische Analyse der Betafaktoren bei faktischen und Vertragskonzernen}

\section{Zusammenfassung}

Im vorliegenden Beitrag werden die Betafaktoren beherrschter Unternehmen bei faktischen und Vertragskonzern-Verhältnissen bis hin zum Squeeze-Out untersucht. Die Ergebnisse zeigen signifikant abnehmende Betafaktoren über verschiedene Konzernstufen. Bei Vertragskonzernen mit Beherrschungs- und Gewinnabführungsvertrag und SqueezeOut-Unternehmen sinken die Betafaktoren der beherrschten Unternehmen auf Werte nahe Null. Die Ergebnisse sind ein Beleg für einen Marktrisiko-Entkopplungseffekt bei Unternehmen, die einem Konzerneinfluss unterliegen. Die Aktien von Minderheitsaktionären beherrschter Unternehmen haben somit im Durchschnitt eine niedrigere Risikoklasse als Aktien von Unternehmen im Streubesitz. Für die Betaschätzung zur Bewertung von Unternehmen mit einem geringen Streubesitzanteil empfehlen wir deshalb, spezielle Regressionsverfahren für unregelmäßig gehandelte Aktien (z.B. Trade-to-Trade-Verfahren) der Verwendung von Peer-Group-Betas vorzuziehen.

\section{Parent Company Control and Market Risk Uncoupling Effect - An empirical analysis of beta factors in factual and contractual groups of companies}

\section{Summary}

This paper examines the estimation of beta factors of dominated firms over different stages of concentrated control, from factual and contractual concern-relationships up to Squeeze-Outs. In our empirical analysis we show a market risk uncoupling effect for firms, which are subject to control by a large shareholder, as evidenced by significantly decreasing beta factors over different stages of control. Firms subject to a profit transfer and domination agreement and Squeeze-Out candidates have beta factors close to zero, and thus belong to a lower risk class than firms with dispersed ownership. These low beta factors can be economically explained by the influence of a controlling shareholder and can be estimated easily, such that they shall be used for the computation of discount factors. Based on our results we suggest preferring the application of thin-trading correction procedures over the use of peer-group betas. 